\title{
Picnodisostosis familiar, reporte de un caso tras 10 años de seguimiento
}

\author{
Cano-Rodríguez AI*
}

Centro Médico ISSEMYM Ecatepec

RESUMEN. Introducción: La picnodisostosis es una rara enfermedad secundaria en una mutación en el gen 1q21 que codifica la catepsina $K$, enzima implicada en el metabolismo de osteonectina, osteopontina y colágeno I. La incidencia mundial es de 1-1.7 casos por millón, sin prevalencia por género, se caracteriza clínicamente por talla baja, deformidades craneales, «cara de pájaro» y fragilidad ósea con tendencia a fracturas patológicas, que afectan predominantemente los huesos largos y ocasionalmente en los pedículos vertebrales. Radiológicamente es característica la presencia de osteoesclerosis con canales medulares permeables. Aunque existen numerosos reportes de casos clínicos en la literatura, pocos son los que describen familias con más de un individuo afectado y el seguimiento suele ser a corto plazo. Objetivo: Analizar la evolución clínica de los pacientes afectados. Material y métodos: Se realizó estudio retrospectivo, descriptivo, observacional de tres pacientes con diagnóstico de picnodisostosis, en el período de Julio 2006 a Marzo de 2016. Resultados: Se observaron diferentes formas de afectación de la picnodisostosis, algunas de ellas atípicas como la espondilólisis y una fractura de escápula en una paciente. Conclusiones: El presente estudio podría ser el análisis longitudinal más extenso del que se tenga registro. Conocer la variedad de manifestaciones y complicaciones presentadas permitirá al lector seleccionar el mejor método de tratamiento para cada caso.

Palabras clave: Picnodisostosis, catepsina K, síndrome de Toulouse-Lautrec.
ABSTRACT. Introduction: Pycnodysostosis is a rare disease secondary to a mutation in gen 1q21 that codifies the cathepsin $K$, proteolitic enzyme implicated in the metabolism of osteonectin, osteopontin and type I colagen. Its global incidence is around 1-1.7 cases per million, without genre prevalences, it is clinically caracterized by short stature, craneal deformities, «bird's face» and bone fragility with pathological fractures tendency predominantly affecting long bones and occasionally vertebral pedicles. Radiologically is characterized by sclerous bones with permeable medular cannel. Despite there are numerous clinical reports on medical literature, just a litlle describe families with more than one afected member and its followship is usually short-term. Objective: To analize clinical evolution of these afected patients. Material and methods: A retrospective, descriptive, observational study was reelized in three patients with diagnosis of pycnodisostosis, between July 2006 and March 2016. Results: Different affection forms of pycnodisostosis where observed, some of them, atipical, as for example spondilolisis and a escapule fracture in one patien. Conclusions: The present study could be the longest longitudinal report ever registered. By knowing the presented variety of manifestations and complications, the reader could select the best treatment method for each case.

Key words: Pycnodysostosis, cathepsin K, Toulouse-Lautrec syndrome.

\footnotetext{
* Servicio de Ortopedia. Centro Médico ISSEMyM Ecatepec.
}

Dirección para correspondencia:

Ángel Ignacio Cano-Rodríguez

E-mail: drangelcanordzl@hotmail.com 


\section{Introducción}

La picnodisostosis es una enfermedad autosómica recesiva rara, producida por una disfunción de los osteoclastos. ${ }^{1}$ El primer caso fue descrito en 1923 por Montanari, pero la enfermedad tal y como se la conoce actualmente fue descrita por Maroteaux y Lamy en $1962,{ }^{2}$ conocido como síndrome de Toulouse-Lautrec por referencia a la afectación que sufría dicho pintor francés, uno de los más importantes exponentes del neoimpresionismo de la segunda mitad del siglo XIX. ${ }^{3}$

Henri Marie Raymond de Toulouse-Lautrec-Monfa, nació el 24 de Noviembre de 1864, en el centro de Albi, en Francia y fue el primogénito de una de las familias aristocráticas más importantes de ese país. A los 14 años de edad sufrió una caída mientras jugaba y a pesar de no ser aparatosa, se fracturó el fémur izquierdo (Figura 1).

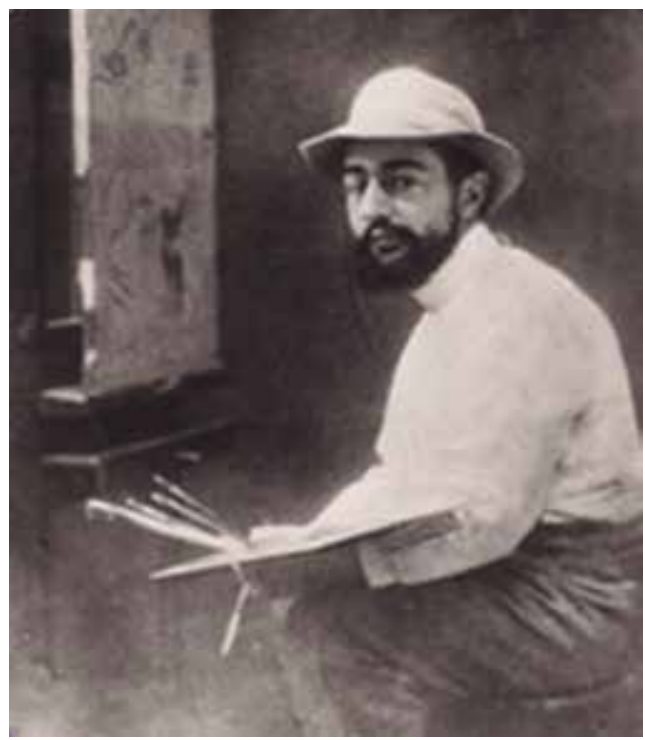

Figura 1: Henri Marie de Toulouse-Lautrec-Monfa.
Durante los dos años posteriores a su fractura permaneció en silla de ruedas y se dedicó a la pintura. Tuvo dos célebres y entrañables amigos: Émile Bernard y Vincent van Gogh. En 1884, se traslada a la localidad parisiense de Montmartre donde comenzó a hundirse en el alcoholismo y contrajo sífilis. Murió a los 37 años a causa de una hemorragia intracerebral, cuyo origen probablemente fue el compromiso meningovascular de la sífilis. ${ }^{3,4}$

Es considerada una patología osteocondensante, junto con la enfermedad de Paget, esclerosteosis, hiperostosis y otras patologías poco frecuentes cuya etiología parece obedecer a polimorfismos genéticos que producen alteraciones en vías de señalización en el osteoclasto, el osteoblasto o en ambas células con desequilibrio en la unidad de remodelado óseo; no obstante, el mecanismo molecular y genético de algunas entidades, permanece en estudio. ${ }^{5}$

Se estima que la prevalencia mundial de un caso por cada millón de habitantes, sin predominio por el género, es debida a una mutación en el gen 1q21, que codifica la catepsina $\mathrm{K}$, enzima implicada en el metabolismo de osteonectina, osteopontina y colágeno $\mathrm{I}^{6}$

Clínicamente se caracteriza por talla baja, acroosteólisis de falanges distales, displasias ungueales, displasia clavicular, deformidades craneales secundarias al retardo en el cierre de suturas y fontanelas; miembros cortos, micrognatia, maxilar superior obtuso e inferior aplanado, lo que confiere al paciente el aspecto de «cara de pájaro», retraso en la aparición de los dientes; y fragilidad ósea con tendencia a las fracturas de los huesos largos. ${ }^{7}$

Radiográficamente hablando, es característica la presencia de osteoesclerosis, con canales medulares permeables. La placa de cráneo, da la apariencia, con los huesos wormianos, de un antifaz; persistencia de fontanelas abiertas, sin compromiso de los agujeros de la base del cráneo; los senos paranasales cerrados. ${ }^{8}$ En columna, falta de segmentación atlantoaxoidea y a nivel lumbosacro, puede haber espondilolistesis, lo cual es sumamente inusual (Tabla 1). ${ }^{9}$

\begin{tabular}{|c|c|c|c|}
\hline Características & Osteopetrosis & Disostosis cleidocraneal & Picnodisostosis \\
\hline Base del cráneo & $\uparrow$ Densidad & Normal o raramente $\uparrow$ & $\uparrow$ Densidad \\
\hline Suturas craneales & Normales & Normales & Abiertas \\
\hline Senos paranasales & Velados & Normales & Velados o cerrados \\
\hline Mandíbula & Normal & Normal & Pérdida del ángulo \\
\hline Clavícula & Presente y normal & Ausencia o displásica & Presente, a veces displásica \\
\hline Manos y pies & Normales & Normales & $\begin{array}{l}\text { Uñas predominantes, falanges cortas, } \\
\text { aplásicas en manojos }\end{array}$ \\
\hline Pelvis & Coxa vara & Normal & Coxa plana \\
\hline Fracturas espontáneas & Presentes & Ausentes & Presentes \\
\hline Textura ósea & $\begin{array}{l}\text { T Densidad con obliteración del } \\
\text { canal intramedular }\end{array}$ & Normal o raramente $\uparrow$ & $\begin{array}{l}\uparrow \text { Densidad con obliteración del canal } \\
\text { intramedular }\end{array}$ \\
\hline Hallazgos sanguíneos & Anemia aplásica & Normal & Normal \\
\hline Genética & $\begin{array}{l}\text { Dominantes: la forma anémica } \\
\text { recesiva }\end{array}$ & Dominante & Recesiva \\
\hline Estatura & Generalmente normal & Generalmente normal & Corta \\
\hline
\end{tabular}


Las fracturas ocurren generalmente después de la segunda década de la vida, el tratamiento puede realizarse con buenos resultados mediante técnicas habituales, ya sean ortopédicas o quirúrgicas. Aunque se debe tener muy en cuenta el diámetro medular cuando se opta por enclavados intramedulares fresados, ya que éste puede ser más estrecho y la cortical aparecer más esclerosa y resistente al paso de las fresas.

Algunos autores han empleado bifosfonatos en el tratamiento de la picnodisostosis; sin embargo, no se dispone de evidencia suficiente que avale su utilización. ${ }^{10}$

\section{Material y métodos}

Se realizó estudio retrospectivo, descriptivo, observacional de tres pacientes con diagnóstico de picnodisostosis, en el período de Julio 2006 a Marzo de 2016.

Descripción del árbol genealógico: se presenta el caso de una familia originaria y residente del Estado de México (Figura 2). Donde los tres hijos padecen picnodisostosis (marcados en color gris). Se sabe que el abuelo tuvo una hija con una pareja previa, pero se desconocen más datos de la misma, incluyendo descendencia. El padre de los pacientes tiene dos hermanos; curiosamente, el mayor de ellos se encuentra casado y tiene cuatro hijos sanos con una de las hermanas mayores de la madre de los pacientes (señaladas en color azul claro).

Una prima paterna (señalada en color azul fuerte), de 16 años, se encuentra en este momento, cursando embarazo de 20 semanas de gestación aproximadamente, con posibilidades de transmitir la enfermedad a la siguiente generación, lo cual sabe que ocurre por rama paterna.

Del lado materno, se incluyen 82 familiares consanguíneos; ninguno de ellos afectado por la entidad, por lo que no se muestra el árbol genealógico.

Caso 1: Mujer de 20 años, a quien identificamos con las iniciales JMFC, producto de la primera gesta, soltera, sin hijos, estudiante de licenciatura. Presentó la primer fractura a la edad de ocho años, siendo ésta diafisiaria de tibia derecha, aproximadamente 18 meses después presenta fractura diafisaria de tibia izquierda. Fractura de quinto metatarsiano izquierdo a los nueve años, fractura de falange no especificada de pie izquierdo a la edad de 11 años. Manejada inicialmente en ortopedia de su hospital zonal. Canalizada para su atención integral a nuestro centro médico, recibiendo su primer consulta en Julio de 2006 a la edad de 11 años, desde entonces se han documentado una variada serie de lesiones: 1. Esclerosis facetaria L4-L5 derecha con callo óseo hipertrófico en la lámina derecha de L5 en Mayo de 2008, a la edad de 13 años. 2. Fractura de tres metatarsianos del pie izquierdo en Octubre de 2008, teniendo la paciente 13 años de edad aún. 3. Fractura diafisiaria de tibia derecha por segunda ocasión en Junio de 2009, a la edad de 14 años. 4. En Septiembre de 2009 se realizó tomografía de control, encontrándose fractura parcialmente remodelada de la lámina y del pedículo L5 derecho con subluxación facetaria L4-L5 izquierda. 5. En Noviembre de 2013, a la edad de 18 años, presenta, por tercera ocasión, fractura diafisiaria de tibia derecha, siendo manejada una vez más con yeso, consolidando en forma aceptable, sin complicaciones ni secuelas (Figura 3).

Caso 2: Mujer de 18 años, producto de la segunda gesta, a quien identificamos con las iniciales LBFC, soltera, sin hijos, estudiante de licenciatura. Debutó con fractura diafisiaria de tibia izquierda a los 10 años, con refractura pocos meses después, siendo manejada con escayola de yeso en su hospital de zona, sin complicaciones ni secuelas. En Octubre de 2009, a la edad de 12 años, se diagnostica fractura de escápula derecha, dándose manejo conservador. Al mes siguiente, Noviembre de 2009, acude a urgencias por presentar fractura espontánea de fémur derecho, realizándose osteosíntesis una semana después con clavos elásticos de titanio, centromedulares (TENS).

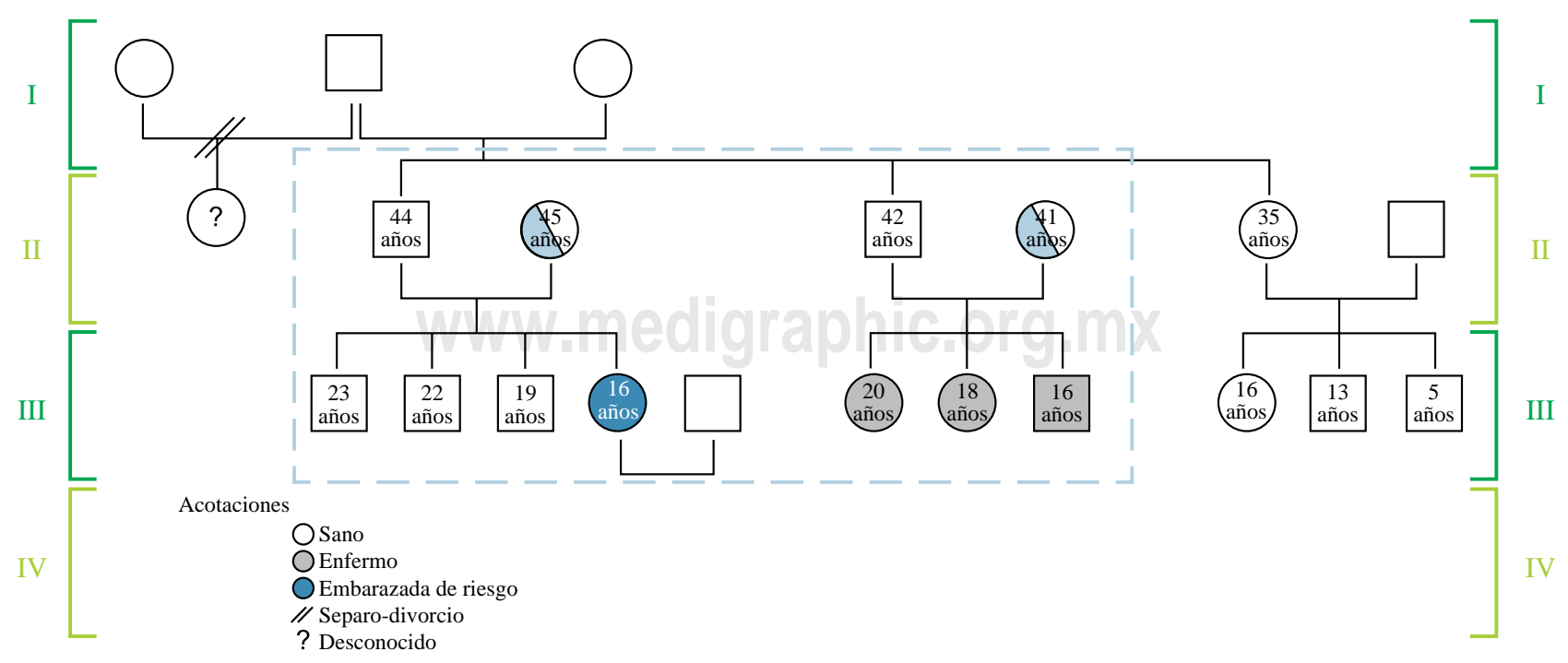

Figura 2: Árbol genealógico, familia paterna. 
Evolucionando en forma insatisfactoria, con retraso en la consolidación y angulación patológica. En Marzo de 2010, a la edad de 13 años, sin haberse conseguido la consolidación del fémur derecho ocurre fractura diafisaria del fémur contralateral. Siendo manejada quirúrgicamente en esa ocasión, se realizó osteosíntesis con placa en ambos fémures. Como complicación presenta en esta ocasión fístula osteocutánea en muslo derecho, detectándose además, nueve meses después, en Enero de 2011, fatiga de material de osteosíntesis, por lo que se decide retiro del mismo. En Mayo de 2014, a la edad de 16 años, presenta, por tercera ocasión fractura diafisaria de tibia izquierda, se le manejó en forma conservadora, sin complicaciones ni secuelas funcionales aunque con angulación anterior (Figura 4).
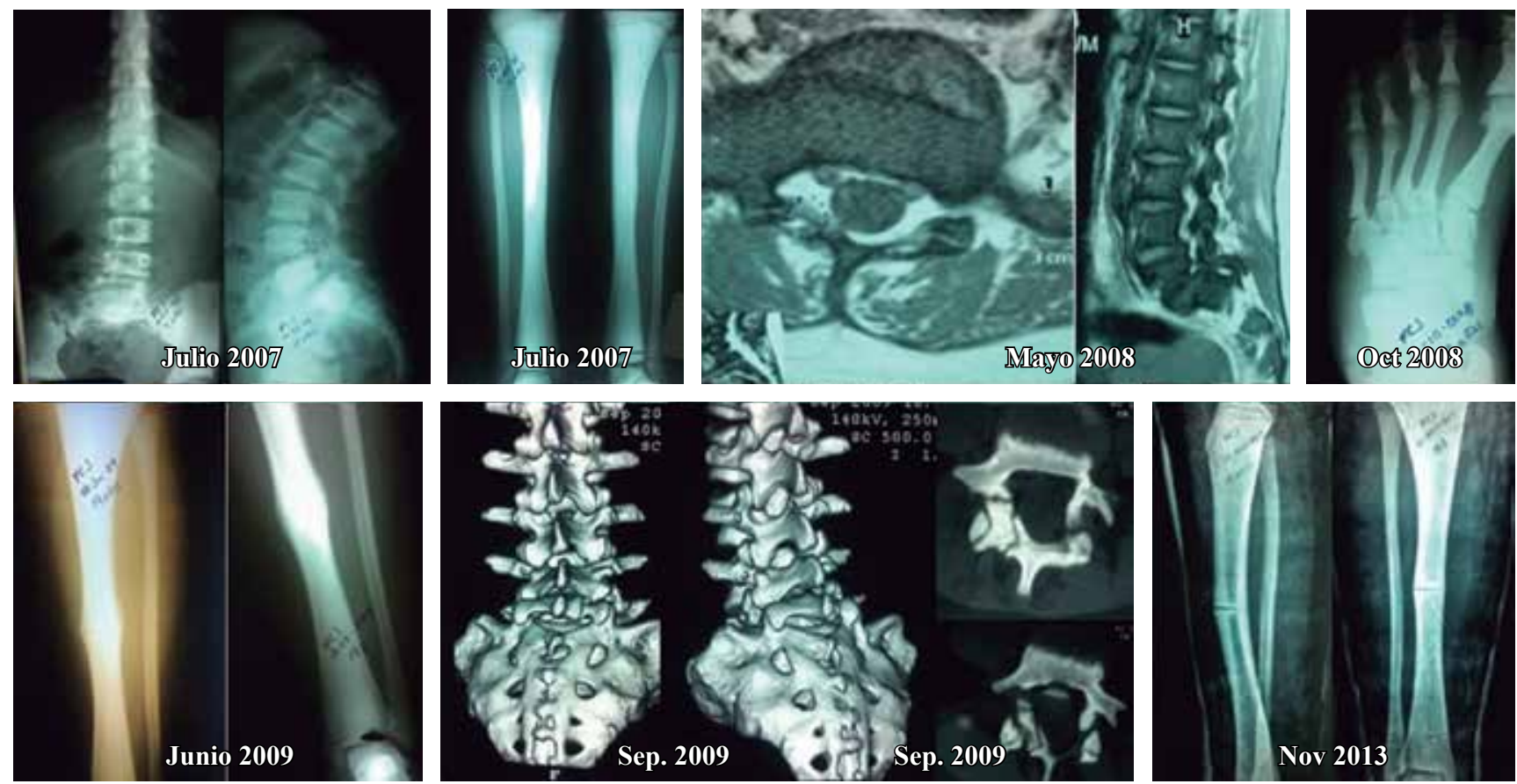

Figura 3: Resumen del expediente radiográfico del caso 1. JMFC. Léase el texto para mayor referencia.
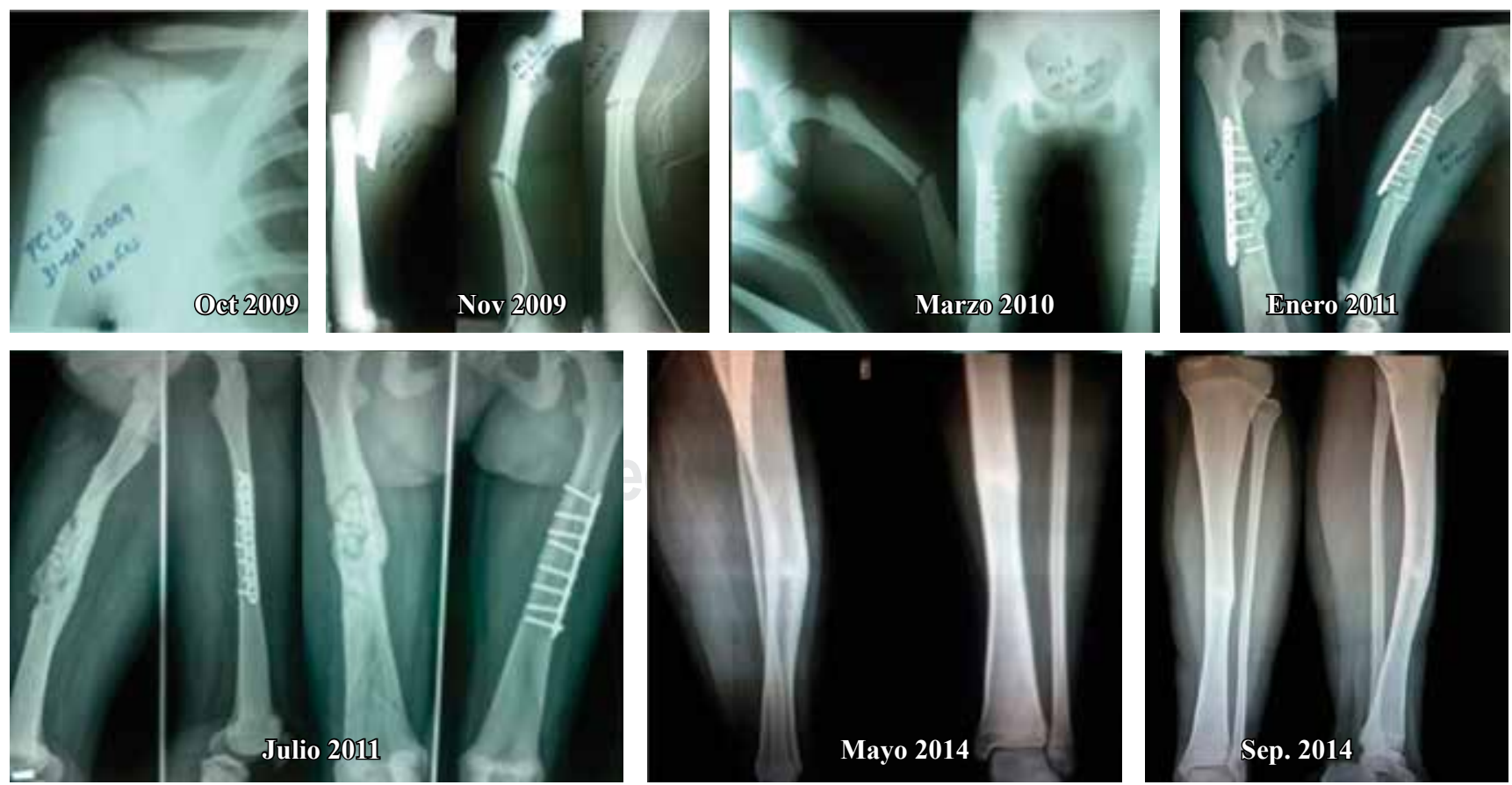

Figura 4: Resumen del expediente radiográfico del caso 2. LBFC. Léase el texto para mayor referencia. 


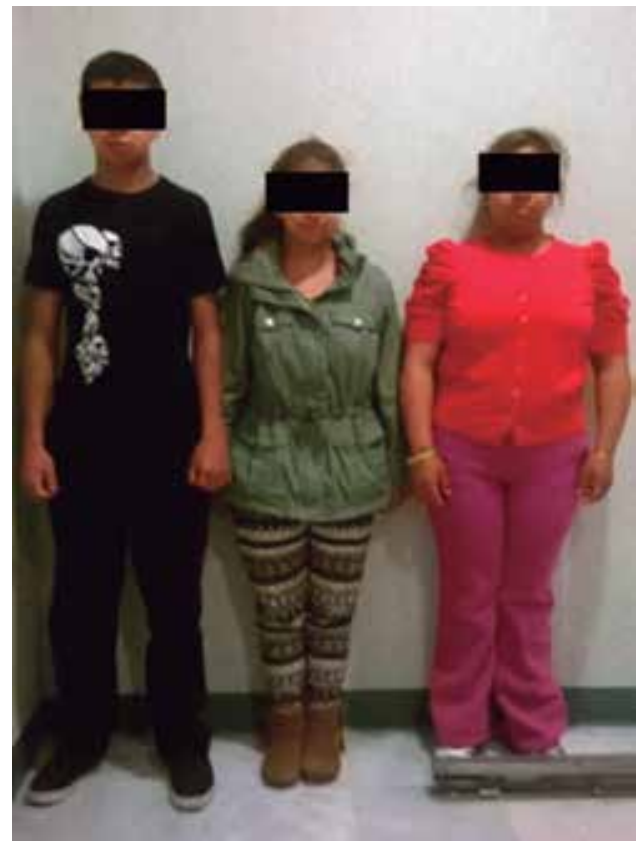

Figura 5: Fotografía casual de la familia. Se les permitió no utilizar bata clínica respetando su preferencia y manteniendo en anonimato su identidad.

Caso 3: Hombre de 16 años, producto de la tercera gesta, a quien identificamos con las iniciales BFC, soltero, sin hijos, estudiante de preparatoria. Jamás ha presentado una fractura a pesar de encontrarse también afectado (Figura 5).

\section{Discusión}

Diversos autores, de todas las naciones, a través de los años, han documentado decenas de casos donde la enfermedad afecta únicamente a un miembro de la familia. ${ }^{1,6,7,8,11}$ En 1992, Thora ${ }^{12}$ y colaboradores documentaron un caso donde, un femenino de 10 años y su hermano, masculino de seis años, productos de padres no consanguíneos, resultaron afectados por la enfermedad, demostrando que no sólo se manifiesta en hijos de padres consanguíneos.

En 2012, Anuradha ${ }^{13}$ y posteriormente Tummala, ${ }^{14}$ en 2013, reportaron casos en los que los hermanos varones de una misma familia presentaban la afección, limitándose únicamente a describir las características morfológicas de los pacientes. Llama la atención en sus publicaciones que solamente los productos masculinos fueron afectados, siendo las hermanas completamente normales.

En la literatura mexicana, se encontró sólo una mención incidental de un caso con cuatro hermanos afectados; pero, de igual manera, la descripción fue somera y enfocada al tratamiento odontológico de uno de ellos. ${ }^{15}$

Pocos autores han documentado seguimientos a mediano plazo, González-Ávila y su grupo documentaron la evolución a dos años del caso de un paciente con fractura femoral. ${ }^{16} \mathrm{El}$ presente artículo, a diferencia de la mayoría de los publicados hasta el momento, documenta secuencialmente la evolución de los pacientes a lo largo de casi 10 años, lo que podría ser el seguimiento más largo jamás registrado de una familia con picnodisostosis.

Asimismo, no es común que se haga un desglose analítico del árbol genealógico de tales pacientes como en las publicaciones de Jáquez, Milles y Marrero-Riverón. ${ }^{17,18,19}$ En esta serie de casos, llama la atención que existen dos uniones entre miembros de ambas familias (dos hermanos de la misma familia paterna con dos hermanas de la misma familia materna), sin que ninguno de los cuatro hijos de la primera unión presentase la afectación, mientras que por el otro lado, los tres hijos de la segunda unión heredaron el padecimiento. No se encuentran registros de un hecho similar en la bibliografía internacional.

Por otra parte, son ocasionales las publicaciones que describen casos de fracturas patológicas en la columna vertebral, como las documentadas en la paciente JMFC (Caso 1)..$^{20,21}$

Este artículo presenta otro hecho no documentado en la bibliografía, la fractura de la escápula derecha de la paciente LBFC (Caso 2); lo habitual en la picnodisostosis es que se fracturen los huesos largos, en su mayoría, de miembros pélvicos, no así huesos planos en miembros torácicos.

\section{Conclusión}

La picnodisostosis es una rara enfermedad con amplia variedad de formas de presentación en todos los segmentos del esqueleto. Muchos casos han sido documentados desde su primera descripción en 1962, siendo diversas las propuestas terapéuticas empleadas y los resultados funcionales.

Consideramos el presente estudio de gran importancia y trascendencia ya que presenta hechos no registrados en la bibliografía internacional que podrían ser útiles en el acervo académico del ortopedista, ya que conocer la variedad de manifestaciones y complicaciones de la picnodisostosis, permitirá al lector seleccionar el mejor método de tratamiento para cada caso.

\section{Bibliografía}

1. Sánchez-Lázaro JA, Linares-Álvarez L. Picnodisostosis: una rara enfermedad con fracturas frecuentes. Semergen. 2014; 40(3): e47-50.

2. Maroteaux P, Lamy M. Pyknodysostosis. Presse Med. 1962; 70: 9991002.

3. Alcalá-Cerra G, Alcalá-Cerra L. Picnodistosis: el caso de ToulouseLautrec. Salud Uninorte. 2006; 22(1): 52-9.

4. Vergara-Amador E. Henri de Toulouse-Lautrec y la picnodisostosis. Rev Col Or Tra. 2008; 22(4): 282-6.

5. Mejía-Vallejo J, Calvo E, Restrepo JF, Iglesias-Gamarra A. Enfermedades osteocondensantes: una nueva visión clínicoradiológica soportada en la genética y la inmunoosteología. Rev Colomb Reumatol. 2009; 16(1): 46-60.

6. Hermosilla-Sánchez F, Renovell-Ferrer P. Picnodisostosis y fragilidad ósea. Caso clínico. Rev Esp Cir Osteoartic. 2008; 236(46): 179-82.

7. Alves N, Oliveira RJ, Deana NF, Sampaio JCA. Morphological features of pycnodysostosis with emphasis on clinical and radiographic maxillofacial findings. Int J Morphol. 2013; 31(3): 921-4.

8. Gómez-Velázquez L. Picnodisostosis: Reporte de un caso. Rev Mex Ortop Ped. 2004; 6(1): 22-24. 
9. Ornetti P, Prati C, Fery-Blanco C, Streit G, Toussirot E, Wendling D. Pedicle stress fracture: an unusual complication of pycnodysostosis. Clin Rheumatol. 2008; 27(3): 385-7.

10. Parrón R, Rivera I, Pajares S, Vicario C, Barriga A. Problemas ortopédicos en la picnodisostosis. Patología del Aparato Locomotor. 2006; 4(1): 70-3.

11. Burguet-i-Arfelis A, Bachiller-Corral FJ, Morales-Piga A. Dentición anómala en niña de 8 años. Reumatol Clin. 2008; 4(5): 207-9.

12. Thora S, et al. Pycnodysostosis. Indian Pediatrics. 1993; 30: 796-800.

13. Anuradha S, Bharathi K. Pycnodysostosis affecting only the male siblings of a family. A rare case report. Int J Pharm Bio Sci. 2012; 3(4): 526-33.

14. Tummala, P, Rao KB, Lakshmi BS. Pycnodysostosis: a case report of radiological analysis. JDMS. 2013; 12(3): 6-8.

15. Gamboa-Mejía K, González-Trejo J, Fragoso-Ríos R. Picnodisostosis. Rev Odont Mex. 2005; 9(2): 92-6.
16. González-Ávila M, Hernández-Mansilla R, Améstica-Lazcano G. Picnodisostosis y fractura femoral: reporte y seguimiento a 2 años. Bol Hosp Viña del Mar. 2014, 70(3): 96-9.

17. Jáquez M, Espinal M, Vélez J. Síndrome de Down en una madre afectada de picnodisostosis. Reporte de ambos casos. Arch Dom Pediatr. 1987; 23(2): 61-5.

18. Mills KL, Johnston AW. Pycnodysostosis. J Med Genet. 1988; 25(8): 550-3.

19. Marrero-Riverón LO, Rondón-García V, Barbán-Lores D, MoralesPeralta E, Quintana-Rodríguez FJ. Estudio en una familia de una paciente con picnodisostosis. Rev Cubana Ortop Traumatol. 2004; 18(1): 34-40.

20. Beguiristain JL, Arriola FJ, Leyes M. Lumbar spine anomalies in a pycnodysostosis case. Eur Spine J. 1995; 4(5): 320-1.

21. Zenke MS, Hatori M, Tago S, Hosaka M, Kokubun S. Pycnodysostosis associated with spondylolysis. Arch Orthop Trauma Surg. 2002; 122(4): 248-50. 